Review

\title{
Digital dementia in the internet generation: excessive screen time during brain development will increase the risk of Alzheimer's disease and related dementias in adulthood
}

\author{
Laurie A. Manwell ${ }^{1, *}$, Merelle Tadros ${ }^{2}$, Tiana M. Ciccarelli ${ }^{2}$, Roelof Eikelboom ${ }^{1}$ \\ ${ }^{1}$ Department of Psychology, Faculty of Science, Wilfrid Laurier University, Waterloo, ON N2L 3C5, Canada \\ ${ }^{2}$ Department of Health Sciences, Faculty of Science, Wilfrid Laurier University, Waterloo, ON N2L 3C5, Canada \\ *Correspondence: Imanwell@wlu.ca; lauriemanwell@gmail.com (Laurie A. Manwell)
}

\section{DOI:10.31083/j.jin2101028}

This is an open access article under the CC BY 4.0 license (https://creativecommons.org/licenses/by/4.0/).

Submitted: 23 July 2021 Revised: 17 August 2021 Accepted: 31 August 2021 Published: 28 January 2022

Converging evidence from biopsychosocial research in humans and animals demonstrates that chronic sensory stimulation (via excessive screen exposure) affects brain development increasing the risk of cognitive, emotional, and behavioural disorders in adolescents and young adults. Emerging evidence suggests that some of these effects are similar to those seen in adults with symptoms of mild cognitive impairment $(\mathrm{MCl})$ in the early stages of dementia, including impaired concentration, orientation, acquisition of recent memories (anterograde amnesia), recall of past memories (retrograde amnesia), social functioning, and self-care. Excessive screen time is known to alter gray matter and white volumes in the brain, increase the risk of mental disorders, and impair acquisition of memories and learning which are known risk factors for dementia. Chronic sensory overstimulation (i.e., excessive screen time) during brain development increases the risk of accelerated neurodegeneration in adulthood (i.e., amnesia, early onset dementia). This relationship is affected by several mediating/moderating factors (e.g., IQ decline, learning impairments and mental illness). We hypothesize that excessive screen exposure during critical periods of development in Generation Z will lead to mild cognitive impairments in early to middle adulthood resulting in substantially increased rates of early onset dementia in later adulthood. We predict that from 2060 to 2100, the rates of Alzheimer's disease and related dementias (ADRD) will increase significantly, far above the Centres for Disease Control (CDC) projected estimates of a two-fold increase, to upwards of a four-to-six-fold increase. The CDC estimates are based entirely on factors related to the age, sex, race and ethnicity of individuals born before 1950 who did not have access to mobile digital technology during critical periods of brain development. Compared to previous generations, the average 17-19-year-old spends approximately 6 hours a day on mobile digital devices (MDD) (smartphones, tablets, and laptop computers) whereas individuals born before 1950 at the same age spent zero. Our estimates include the documented effects of excessive screen time on individuals born after 1980, Millennials and Generation Z, who will be the majority of individuals $\geq 65$ years old. An estimated 4-to6-fold increase in rates of ADRD post-2060 will result in widespread societal and economic distress and the complete collapse of already overburdened healthcare systems in developed countries. Preventative measures must be set in place immediately including investments and interventions in public education, social policy, laws, and healthcare.

\section{Keywords}

Excessive screen time; Brain development; Alzheimer's disease and related dementias (ADRD); Mild cognitive impairment (MCI); Internet generation (iCen) or Generation Z; Anterograde amnesia

\section{Introduction}

Researchers at the Centers for Disease Control and Prevention (CDC) project that the rate of Alzheimer's disease and related dementias (ADRD) in the United States will increase 2 -fold, from $1.6 \%$ to $3.3 \%$, by 2060 [1]. These estimates are based on data for the ADRD burden of disease in 2014 for adults aged $\geq 65$ years according to age, sex, race, and ethnicity factors which primarily represents individuals born between 1925-1945 (Silent Generation) and 1946-1964 (Baby Boomers) [1]. These factors do not account for significant and relevant differences between individuals born before 1965 and those born after 1980 (Millennials) and 1995 (Generation $\mathrm{Z}$ ). The CDC estimates are based upon factors currently known about the Silent Generation and Baby Boomers who are aged $\geq 65$ years today, whereas most of the population aged $\geq 65$ years older in 2060 will be Millennials and Generation Z. There are at least two known critical factors that differ significantly between those born before 1965 and those born after 1980 which are related to the risk of neurodegenerative disorders such as ADRD: these are overall intelligence level (i.e., assessed by IQ testing) and excessive screen time (i.e., time spent on electronic media).

Growing evidence documents a negative (or reverse) Flynn Effect, which is a progressive decline in population intelligence (i.e., intelligence quotient or IQ test scores), on a global scale starting around 1975 and projected to continue into 2050 [2]. The projected global decline in intelligence of approximately 1.28 I.Q. points for $2000-2050$ is thought to be related to environmental rather than genetic causes and to have started with Millennials and will include Generation $\mathrm{Z}$ [3]. Converging evidence shows that excessive screen time (i.e., more than 2-3 h/day exposure to electronic media including television, computers, and mobile devices such as 


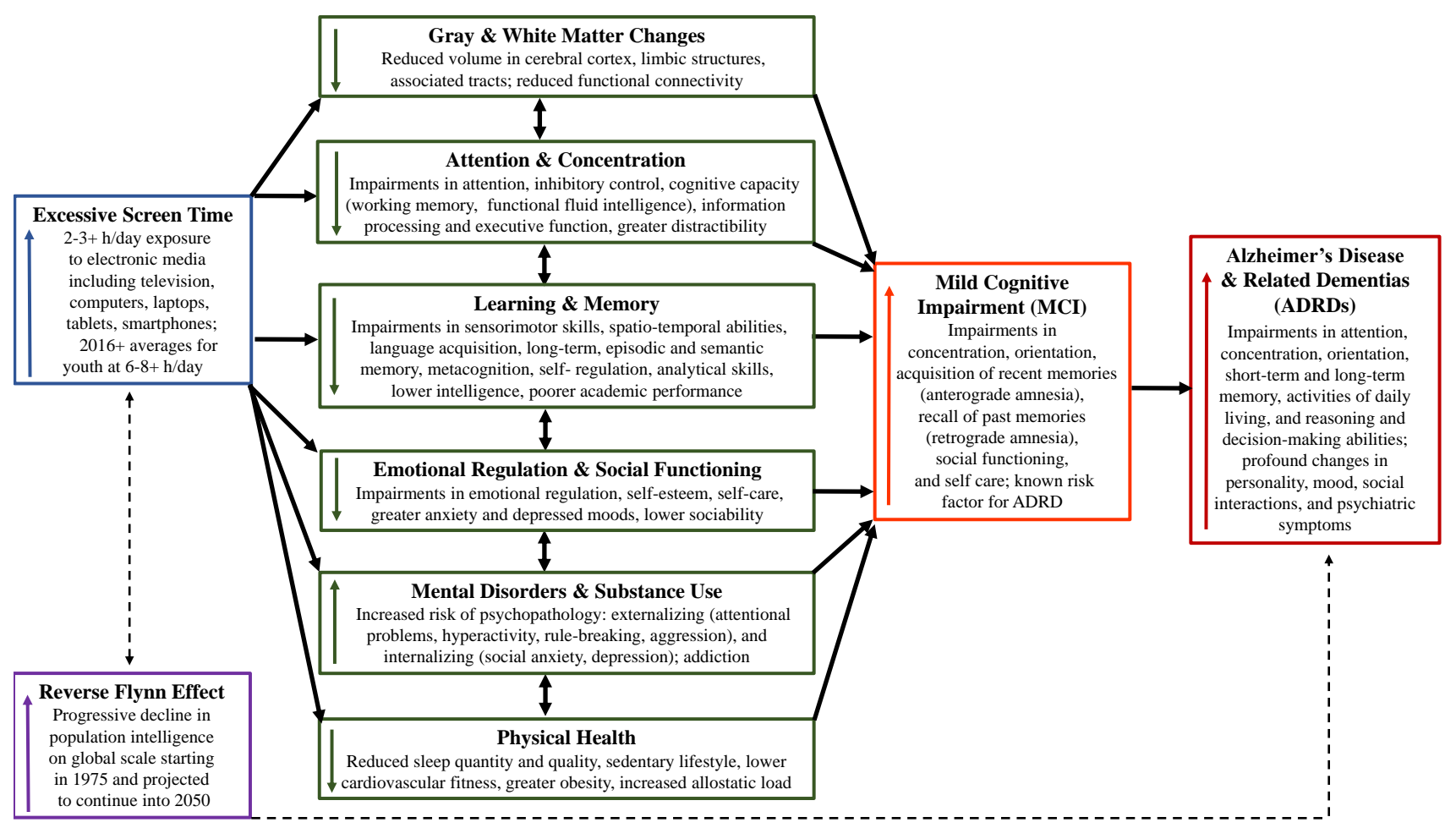

Fig. 1. Schematic model of the effects of excessive screen time on various factors that contribute to the development of mild cognitive impairment and dementia. Excessive screen time (i.e., $>2-3 \mathrm{~h}$ /day exposure to electronic media including television, computers, and mobile electronic devices) has been shown to affect brain development (i.e., structure and function) and increase the risk of cognitive, emotional, and behavioural disorders in adolescents and young adults by negatively impacting attention and concentration, learning and memory, emotional regulation and social functioning, physical health, and development of mental disorders and substance use. These effects are similar to the symptoms of mild cognitive impairment (MCI) seen in older adults that increase the risk of Alzheimer's disease and Related Dementias (ADRDs). The current decline in global IQ, estimated to have started post-1975 and projected to continue into 2050, parallels significant population level increases in screen time and could also increase the risk of MCI and ADRD. Solid lines represent empirically-supported connections between factors and dotted lines represent theoretically-supported connections.

smartphones, tablets, and laptops), particularly during brain development, is related to increased risk of learning and memory impairments, attentional and emotional disorders, substance abuse, and changes in cortical gray and white matter volumes [4]. These observations are consistent with the cognitive-behavioural-brain reserve (CBBR) hypothesis of dementia, which suggests that more complex patterns of neural and mental activity in early, middle, and later life stages are associated with decreased risk of dementia, whereas less complex patterns are associated with an increased risk of dementia [5]. We propose that these two factors, declining intelligence and excessive screen time, which came to prominence for Millennials and Generation Z, will compound over time reducing the overall cognitive-behavioural-brain reserve of these individuals significantly increasing their risk of ADRD. We predict that from 2060 to 2100, the rates of ADRD will rise far above the CDC projected estimates up to a four- to six-fold increase. The CDC estimates are based entirely upon factors related to the age, sex, race and ethnicity of individuals born before 1950 who did not have access to mobile digital technology during critical periods of brain development. Our estimates include the documented effects of global declines in intelligence levels and excessive screen time for individu- als born after 1980, Millennials and Generation Z, who will comprise most individuals $\geq 65$ years old in 2060 .

\section{Theory}

Converging evidence from biopsychosocial research in humans and in animal models demonstrates that chronic sensory stimulation via excessive screen time (i.e., defined as more than $2-3 \mathrm{~h}$ /day exposure to electronic media including television, computers, and mobile electronic devices) affects brain development, increasing the risk of cognitive, emotional, and behavioural disorders in adolescents and young adults [4]. Excessive screen time negatively impacts learning [6-8], memory [9-11], attention [12-15], concentration $[12,13,16]$, emotional regulation and social functioning [1721]. Effects of excessive screen time appear similar to symptoms of mild cognitive impairment (MCI) seen in adults in the early stages of dementia, including impaired concentration, orientation, acquisition of recent memories (anterograde amnesia), recall of memories (retrograde amnesia), social functioning, and self-care $[4,22,23]$. Excessive screen time is also known to increase the risk of mental disorders $[20,24]$, and substance use $[25,26]$ which are known risk factors for dementia $[27,28]$. The current decline in global IQ, 


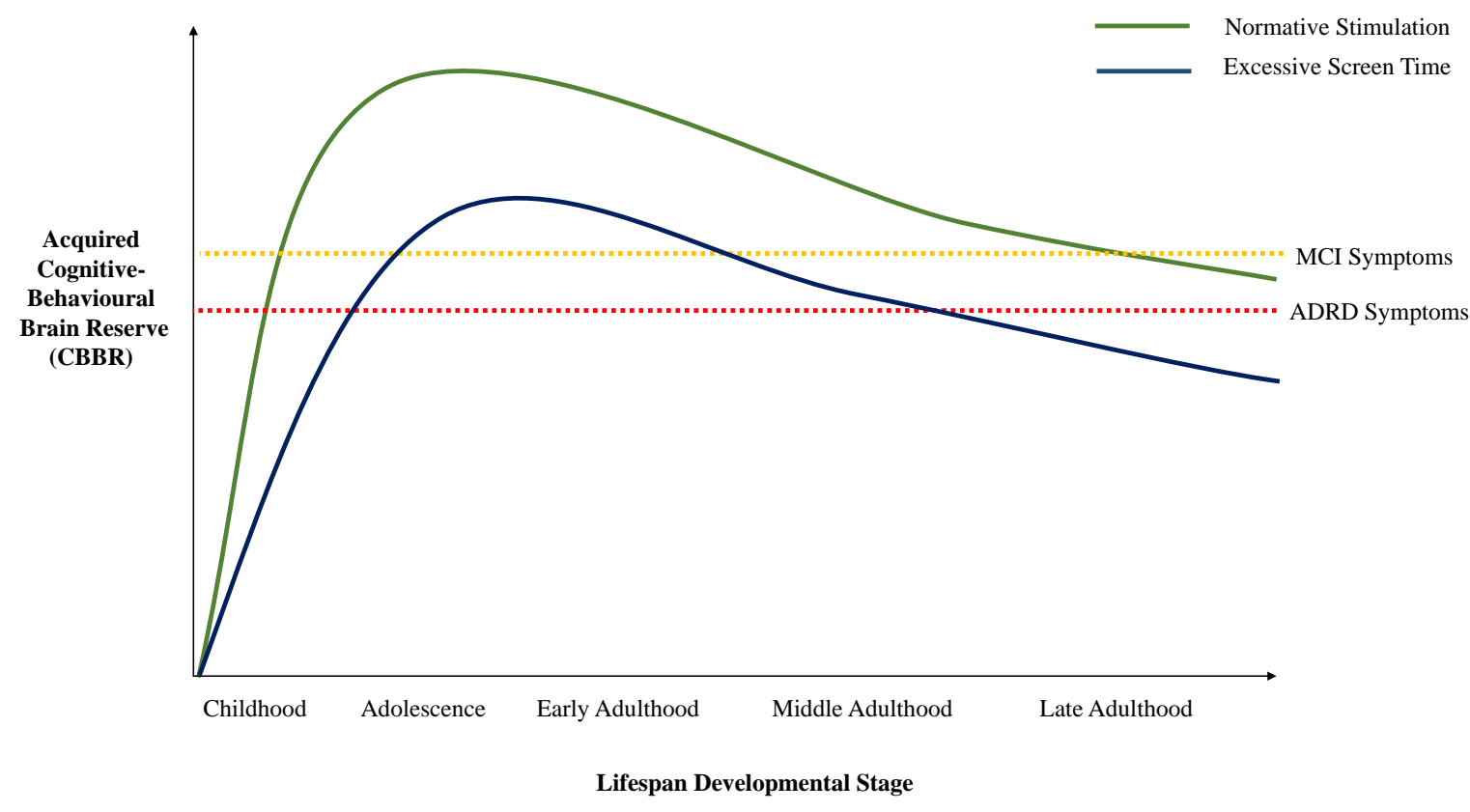

Fig. 2. Schematic model of the effects of excessive screen time on cognitive-behavioural brain reserve and implications for early onset of mild cognitive impairment and dementia. The cognitive-behavioural-brain reserve (CBBR) hypothesis of dementia postulates that more complex patterns of neural and mental activity in early, middle, and later life stages are associated with decreased risk of dementia as opposed to less complex patterns which are associated with increased risks. Brain development occurs in stages marked by periods of massive neuroplasticity (i.e., significant changes in gray and white matter) that correspond to cognitive-behavioural maturation. Neuroimaging studies of connectivity in the brain suggest several dynamic brain networks governing executive functions, intelligence, and social-emotional behaviour emerge early in brain development, increase their functional interactions during adolescence, and variations in patterns of connectivity can predict healthy and pathological trajectories of development into adulthood. Studies show that these cortical networks can be influenced by early environmental experiences including excessive screen time. If the neural circuits underlying these cognitivebehavioural abilities essential for general intelligence and lifetime adaptability are under- or abnormally-developed before adulthood, then it is likely that these changes will persist into early and middle adulthood and be more vulnerable to accelerated neurodegeneration in late adulthood therefore increasing the risk of early onset MCI and ADRDs.

estimated to have started post-1975 and projected to continue into 2050, could contribute to an overall cognitive decline in the world's population increasing the risk of ADRD in individuals $\geq 65$ years old in 2060 and beyond. It is estimated that these two factors, increasing rates of excessive screen time and declining IQ levels, will compound over time to reduce the overall cognitive-behavioural-brain reserve capacity for individuals born after 1975 and increase their rates of ADRD compared to those born before 1950 (refer to Figs. 1,2 and Table 1, Ref. [3, 4, 7-14, 16-21, 23, 25, 29-44]). Specifically, we predict that Millennials and Generation $\mathrm{Z}$ will have a 4to 6-fold increase in ADRD in 2060 compared to recent estimates of ADRD in 2014 for the Silent Generation and Baby Boomers.

\section{Hypothesis}

We hypothesize that for individuals born after 1975, the effects of excessive screen exposure during critical periods of brain development and the global decline in IQ levels will compound over time leading to lower overall cognitivebehavioural-brain reserve. This will translate into a higher prevalence of MCI in early to middle adulthood resulting in substantially increased rates of ADRD in late adulthood. We predict that the rates of ADRD will rise far above the projected estimates from the CDC of a 2-fold increase from 2014 levels [1] up to a 4- to 6-fold increase in 2060 and beyond.

\section{Evaluation of hypothesis 4.1 Critique of CDC estimates}

Using data from the Centers for Medicare and Medicaid Services (CMS) and the United States (US) Census Bureau, Matthews and colleagues [1] estimated a 178\% increase from 2014 to 2060 for the number of Americans projected to have ADRD. This reflects a growth in both aging and minority populations and assumes trends in 2014 will remain constant [1]. Matthews and colleagues [1] suggest that since their estimates are based solely upon unchangeable risk factors for ADRD, other modifiable factors such as educational attainment which tend to have protective effects against dementias may result in an overestimate for future decades. We will argue that not only is the Matthews and colleagues [1] projection not an overestimate, but it is a significant underestimate based upon additional data showing several critical differences between populations aged $\geq 65$ in 2014 compared 
Table 1. Effects of excessive screen time on cognitive-behavioural brain reserve early in life and implications for risk of MCI and ADRD.

Category Effects on Brain, Behaviour \& Cognition (CBBR) Implications for risk of MCI \& ADRD References

Brain structure and Screen time associated with changes in occipital cortex (reduced volume, thinning, sul- Early volume losses in areas governing sensorimotor functios- He et al. [29] (2017); Hong et al. [30, 31]

function cal depth), prefrontal cortex (smaller orbitofrontal volume, thinning), temporal cortex ning, executive functioning, and reward-learning areas could re- (2013); Lee et al. [32] (2018); Paulus et al. [33] (thinning), insula (reduced volume), limbic structures (smaller hippocampus, amygdala, duce overall CBBR and increase risk of cognitive-behavioural (2019); Weng et al. [34] (2013); Yuan et al. ventral striatum), reduced functional connectivity in cortico-subcortical circuits, and mi- disorders including substance use disorders and thus increase risk $[35,36](2011,2013)$ crostructure abnormalities in gray and white matter of MCI and ADRD later in life

Attention and concen- Screen time associated with impairments in attention during development, cognitive ca- Impairments in attention, concentration, and cognitive capacity Carrier et al. [14] (2009); Christakis et al. [12] tration pacity (working memory and functional fluid intelligence), vulnerability to distractions, similar to symptoms of MCI; if occurring early in life, these could (2004); Christakis et al. [13] (2018); Loh \& poorer inhibition, reduced executive control (focused and sustained attention), shallow reduce overall CBBR, adult IQ, and lifetime educational attain- Kanai [37] (2016); Ward et al. [16] (2017) information processing, and poorer academic progress ment which are known risk factors for MCI and ADRD

Learning and memory Screen time associated with impaired development of sensorimotor skills, spatio-temporal Impairments in learning and memory early in life could re- Glass \& Kang [9] (2018); Madigan et al. [7] abilities, problem solving and language acquisition, lower crystalized and fluid intelli- duce overall CBBR, adult IQ and lifetime educational attainment (2019); Mangen et al. [8] (2013); Paulus et al. gence, poorer vocabulary and reading comprehension, reduced metacognitive and self- which are known risk factors for MCI and ADRD; impairments [33] (2019); Sparrow et al. [10] (2011); Tamir regulation skills, and superficial effort and retention of information, poorer long-term in episodic and semantic memory in young adults likely to per- et al. [11] (2018) memory, cognitive development (analytical thinking), and academic performance sist into middle and late adulthood increasing risk of early onset of MCI and ADRD symptoms

Emotional regulation Screen time associated with poorer emotional regulation, inability to stay calm, rein- Symptoms of poor emotional regulation, self-care, and social Boers et al. [17] (2019); Hunt et al. [38] (2018); and socia functioning forcing cognitive-emotional spirals, lower self-esteem, increased anxiety and depressed functioning are characteristic of MCI which is a known risk fac- McNicol \& Thorsteinsson [23] (2017); NeOmoods, lower productivity and curiosity, fewer social interactions, lower sociability, up- tor for ADRD wards social comparison, uncooperative attitudes and behaviour, sexual activity phytou et al. [4] (2019); Twenge \& Campbell [20] (2018) Twenge et al. [21] (2019)

Mental disorders and Screen time associated with poorer mental health, overall risk of psychiatric conditions Psychiatric conditions are known risk factors for MCI and Maras et al. [19] (2015); Neophytou et al. [4] substance use including externalizing psychopathology (e. attentional problems, hyperactivity, rule ADRD; chronic exposure to excessive audiovisual stimulation (2019); Yen et al. [39] (2009); He et al. [29] breaking and aggressive behaviour) and internalizing psychopathology (e.g., social anxi- may affect the neuroimmunoendocrine system increasing allo- (2017); Gommans et al. [25] (2015); Twenge ety, depression) and excess behaviour related to reward-learning disruptions (e.g., impulse static load and risk of mental disease \& Campbell [20] (2018) control and addictive behaviours related to the internet and substances)

Physical health Screen time associated with poorer sleep, more sedentary lifestyle, reduced physical activ- Poor physical health is a known risk factor for MCI and ADRD Martin [40] (2011); Twenge et al. [41] (2017); ity, lower cardiovascular fitness, increased incidence of being overweight or obese; may Wethington et al. [42] (2013) directly and indirectly affect the neuroimmunoendocrine system increasing allostatic load and increase risk of physical disease

Global intelligence Significant population level increases in screen time align with significant population level Lower IQ and educational attainment tend to be risk factors for Bratsberg \& Rogeber [3] (2018); Twenge et al. declines in intelligence despite population level increases in educational attainment (i.e., MCI and ADRD Reverse Flynn Effect) 
to 2060. These additional factors include a decline in intelligence [3], despite greater educational attainment [43], and an increase in screen time [44], due to greater access to mobile digital devices, both of which occurred post-1975. Converging research suggests that the negative effects of excessive screen time include impairments in learning, memory, attention, concentration, emotional regulation, and social functioning in young adults that are similar to symptoms of MCI observed in older adults and that typically precede the development of ADRD. MCI is a diagnostic category thought to be a transitional stage from normal to pathological aging, and is characterized by the following minimal impairments to instrumental activities of daily living in the absence of dementia: complaint or history of cognitive decline/impairment, objective evidence of impairment in attention, memory, language, visuospatial skills or executive function, and impairments in normal functional activities [45].

4.2 Neurological and cognitive-behavioural markers of MCI and $A D R D$

ADRDs are progressive brain disorders characterized by gradual and increasing impairments in attention, concentration, and orientation, short-term and long-term memory, activities of daily living, and reasoning and decision making abilities [22, 46]. At the end stages of ADRD, individuals are usually non-verbal, non-ambulatory and unresponsive to their surroundings [46]. The progression from early to end stages of ADRD also include profound changes in personality, mood and social interactions, including anterograde amnesia (i.e., inability to acquire new memories), retrograde amnesia (i.e., trouble recalling past memories), and even psychiatric symptoms such as delusions and hallucinations [46, 47]. Those with ADRD also show poorer social functioning and self-care routines, score higher on tests of anxiety, and have greater sleep disturbances $[48,49]$.

Studies show that regional and global cerebral atrophy rates are well correlated with progressive cognitivebehavioural declines characteristic of Alzheimer's disease (AD) [50-55]. Progressive cerebral atrophy is associated with the stages of AD $[52,54]$. Pre-symptomatic and mildly affected individuals show significant volume loss in the hippocampus and posterior cingulate and neocortical temporoparietal cortices; in contrast, moderately and severely symptomatic individuals show more widespread cerebral atrophy, particularly in the inferolateral areas and frontal lobes with some sparing of the primary motor and sensory areas and cerebellum [52, 54]. Volumetric changes in the hippocampus, entorhinal cortex, whole brain, and ventricles predicted conversions from preclinical (i.e., no symptoms) to clinical stages of MCI or AD and from MCI to AD and were more reliable predictors than standard psychometric measures [53]. For individuals over age 60, gray matter volume decreases of approximately $2 \%$ per year are seen in patients with AD compared to controls [50] and significant losses in white matter essential for communication between brain regions [30, 34, 35, 51, 56]. Reduced cortical thick- ness in the posterior cingulate gyrus is characteristic of $\mathrm{AD}$ in both typical and atypical clinical presentations [57]. These findings demonstrate that cerebral volume is a robust indicator of MCI and ADRD in middle to late adulthood. Factors that may increase cerebral volume loss in early adulthood, or even prevent cerebral volume growth in childhood and adolescence, may greatly accelerate the cognitive-behavioural signs of neurodegeneration in middle adulthood and significantly increase the rates of MCI and ADRD. We will argue that excessive screen time in childhood, adolescence, and early adulthood impairs brain development leading to reduced overall CBBR before middle adulthood increasing the risk of neurodegeneration potentially 10 to 20 years earlier than expected.

\subsection{Global declines in intelligence may increase risk of MCI and dementia in 2060}

The Flynn effect refers to the increasing performance over time on intelligence tests across the population throughout the 20th century [3,58-61]. Studies show increases of approximately 2.50 to 3.00 IQ points per decade with proposed explanations focusing on biological factors (e.g., nutrition, pathogen stress, fertility) and environmental factors (e.g., education, family size, technology, test-taking behaviour) and the interactions between them, referred to as 'social multipliers' (i.e., any environmental factor that confers an advantage improving test performance) [62]. Analyses show substantial gains in fluid IQ (i.e., reasoning-based performance) that are greater than gains for crystallized IQ (i.e., knowledge-based performance), greater gains for adults than children, and positive correlations with economic gains (i.e., GPD growth) [62]. Results also show a deceleration or reversal of gains in recent decades [62].

The reversal of this trend in IQ gains, referred to as the negative Flynn effect, has been documented in several countries in post-1975 birth cohorts [2, 3, 58, 62]. The main contributors to the Flynn effect are thought to be environmental and generate within-family variation based upon exposure differences (e.g., age at exposure, exposure duration), although the specific mechanisms underlying these environmental effects are unclear [3]. The negative Flynn effect may reflect a ceiling effect for environmental advantages (e.g., saturation or diminishing returns) or increases in social multipliers detrimental to IQ (e.g., dysgenic fertility, immigration, declining educational standards) [3, 62]. Although exposure to technology is postulated to positively affect IQ by providing greater visual stimulation, evidence does not support a link between increased fluid IQ and frequency of visual media exposure [62]. In fact, evidence is emerging that excessive screen time may be a significant negative social multiplier contributing to the deceleration and decline of population IQ. The negative Flynn effect starting post-1975 is paralleled by a decline in vocabulary, documented from 1974 to 2016 for American adults; this is despite an increase in educational attainment (e.g., 11.83 to 13.68 years of school completed), which is generally correlated with verbal intel- 
ligence [43]. During this same time period, there has been a significant decrease in reading print (e.g., books, magazines, newspapers) and a critical increase in digital screen exposure (e.g., television, computers, tablets, smartphones) [44]. In a study examining reading comprehension, it was found that students who read from print scored significantly higher on reading comprehension tests than those who read the text digitally [8]. Several explanations were proposed: (i) navigation via scrolling on screens is known to negatively interfere with mental representations of text, (ii) multitasking on a computer (e.g., switching screens to read or answer questions) has hidden cognitive processing and memory costs (e.g., increasing challenges of dividing attentional resources), and (iii) metacognitive and self-regulation skills (i.e., ability to monitor and adapt one's cognitive performance to a task) may have been engaged more with print media, traditionally used for effortful learning, compared to digital media, often used for quick and superficial information gathering [8]. Evidence suggests there are detrimental consequences of excessive screen time for attention, learning, memory, and language development [4]. Research shows that screen time is increasingly replacing time spent on academics for adolescent and young adult students [43, 44, 62] and constant use of digital devices is associated with worse reading comprehension, word reading and vocabulary [8], which may impact overall IQ levels.

\subsection{Significant differences in screen time exposure for individuals} born before and after 1975

The amount of screen time exposure during critical periods of brain development differs significantly for the Silent Generation (born 1925 to 1945), Baby Boomers (born 1946 to 1964), and Generation X (born 1965 to 1979) compared to Millennials (born 1980 to 1995) and Generation Z (born 1996 to 2012). Compared to previous generations, the average 1719 -year-old spends approximately 6 hours a day on mobile digital devices (MDD) (e.g., smartphones, tablets, and laptop computers) [44] whereas individuals born before 1965, the Silent Generation and Baby Boomers, at the same age spent zero hours on MDD. Generation $\mathrm{Z}$ is the first in history to never know a world without the Internet; Generation $\mathrm{Z}$ surpasses passes Millennials on social media use by almost 3 hours each day [63].

Adolescent screen time use has changed dramatically from 2.5 hours a day of TV viewing in 1970 to almost 8 hours a day of TV viewing, internet use, texting, and social media use in 2016 [44]. This represents at least a 3-fold increase in screen use for adolescents as these numbers were reported for leisure time and do not include screen use at work, school or studying [44]. Even the time spent viewing television may be fundamentally different across cohorts as Generation $\mathrm{Z}$ increasingly engages in multiscreen use [6468] which is known to have negative effects on cognitive processing including attention, memory, learning, and comprehension [69-73]. Overall, Generation $\mathrm{Z}$ spends more time online and texting and less time with more traditional media such as magazines, books, and television than previous generations [44].

4.5 The effects of screen time on brain development and implications for cognitive-behavioural-brain reserve in adulthood

Excessive screen time affects brain structure and function, particularly during development, which greatly impacts attentional and inhibitory control, focused concentration, learning, memory, reasoning, and creativity [10, 7476]. These basic capacities are essential for executive control in goal-directed and decision-making behaviours which underlie human intelligence and the essential "ability to adapt to uncertain, changing, and open-ended environments" [74] (pp. 1). If the neural circuits underlying these cognitivebehavioural abilities essential for general intelligence and lifetime adaptability are under- or abnormally-developed before adulthood, then it is likely that these changes will persist into early and middle adulthood and be more vulnerable to accelerated neurodegeneration in late adulthood. Brain development occurs in stages and is marked by periods of massive plasticity (i.e., major neuronal and synaptic rearrangement), particularly the perinatal and periadolescent transitions, the latter of which is characterized by significant gray matter reductions and white matter increases that correspond to cognitive-behavioural maturation [77-79]. Neuroimaging studies of connectivity in the brain suggest several dynamic brain networks governing executive functions, intelligence, and social-emotional behaviour emerge early in brain development, increase their functional interactions during adolescence, and variations in patterns of connectivity can predict healthy and pathological trajectories of development into adulthood [22, 80-82]. Changes in these cortical networks are thought to be influenced by early environmental experiences, including childhood abuse [83], urban upbringing [84], and screen time [33], and may have serious lifetime impacts on general cognitive ability, social-emotional behaviour, psychopathology, and substance use and abuse. Studies show that abnormal patterns of regional connectivity, specifically in frontal and parietal lobe connections, are also found in neurodegenerative conditions including ADRDs, particularly frontotemporal dementia and AD [22].

Preliminary data from The Adolescent Brain and Cognitive Development (ABCD) Study, a multi-site longitudinal neuroimaging study, shows the significant impact that screen time has on the relationship between cognitive-behavioural maturational markers and normal patterns of brain development in children and adolescents $[33,85]$. The study identified several associations between screen time, brain structure (e.g., cortical thickness, gray matter volume, and sulcal depth), and cognition (e.g., fluid and crystallized intelligence) and mental health (e.g., internalizing and externalizing psychopathologies) [33]. First, results showed a robust association between screen time and changes in visual system structural patterns (i.e., reduced volume, thinner cortex, variations in sulcal depth), which may negatively affect the functioning of other cortical networks, such as cognitive 
control regions, that interact with sensorimotor areas [33]. Second, results showed a strong association between screen time, externalizing (but not internalizing) psychopathology, and changes in cortical and limbic structures (e.g., smaller orbitofrontal volume, thinner occipital and temporal cortices, thinner hippocampi) [33], which may help explain the concomitant rise in screen time and disorders of attention and hyperactivity (reviewed in [4]). Third, results showed a more complex pattern of association between screen time, particularly type (e.g., gaming versus social media), and brain structure changes and measures of fluid and crystallized intelligence. For fluid intelligence, gaming activity showed positive associations with prefrontal cortex thinning, whereas social media activity showed negative associations with hippocampal thickness and temporal lobe volume [33]. In contrast, for crystallized intelligence, screen time in general and social media activity showed strong negative associations with occipital and orbitofrontal volume [33]. In a study of young adults, it was demonstrated that excessive social media use is associated with gray matter volume reduction in the bilateral amygdala and right ventral striatum but not the prefrontal cortex [29], suggesting that excess behaviour arises from a developmental disruption of reward-learning [29].

Taken together, these results contribute to evidence from brain imaging and behaviour studies that support the dual system theory of excess problematic behavior [86] which proposes that the development of these abnormal behaviors arises from imbalances between the reward system (mesolimbic dopamine amygdalastriatal) which becomes hyperactive and the inhibition system (prefrontal regions) which becomes hypo-active [29]. Such imbalances have important implications for focusing attention, learning, memory, intelligence, social-emotional regulation, and behavioural addictions. Based on models of drug, alcohol, and gambling addictions, characteristic traits of internet addiction include fixation with playing online games, repeated failed attempts at cessation, increased negative affect, neglecting significant relationships or activities, and the development of tolerance and withdrawal symptoms [30, 35, 36]. Studies show internet addiction is associated with important changes in both gray and white matter in the developing brain, including volumetric loss and functional impairments related to behavioural abnormalities (e.g., impaired cognitive ability and control) [30, 34-36, 87]. For example, individuals with internet addiction showed abnormalities in cortical thickness in several areas involved in cognitive control including the frontal lobe, left lateral orbitofrontal cortex, insula cortex, lingual gyrus, right postcentral gyrus, entorhinal cortex, and inferior parietal cortex [35]. The frontal lobe performs executive functions, such as planning and organizing, and undergoes massive changes from puberty until mid-twenties [74]. The frontal lobe along with volume loss in the striatum, which includes the reward pathway and suppression of impulses, is seen to be affected by gray matter atrophy and white matter volumes; those with gaming addiction showed significant gray matter atrophy in the right orbitofrontal cortex, bilateral insula, and right supplementary motor area [34]. A functional magnetic resonance imaging study found that there is less efficient information processing and a reduced impulse inhibition in those with an internet addiction disorder, along with an increased sensitivity to rewards and an insensitivity to loss was exhibited $[88,89]$. It was identified that those with an internet addiction disorder had abnormal spontaneous brain activity that correlated with poor task performance [35].

Thus, these studies suggest that early reductions in brain volume and functional capacity during brain development may increase the risk of MCI and ADRD which are characterized by significant white and gray matter loss and a deterioration in instrumental activities of daily living. This is consistent with the cognitive-behavioural-brain reserve (CBBR) hypothesis of dementia. Studies show that brain reserve is positively correlated with IQ, educational attainment, and engagement in cognitively stimulating occupational and leisurely activities, and negatively correlated with the transition from MCI to dementia $[5,90]$.

\subsection{Main developmental effects of screen time on learning and memory}

The term digital dementia was first coined by the neuroscientist Manfred Spitzer to refer to the literal 'minddeteriorating' effects of digital technology; specifically, that excessive use of digital devices is associated with cognitive impairments characteristic of dementia, such as decreased attention and impaired memory, and these effects are being seen increasingly in younger adults not expected to be experiencing neurodegeneration characteristic of old age [91-93]. For example, Generation $\mathrm{Z}$ children began regularly watching television by 3 months of age [94] compared to 4 years of age for Generation X children [95]. Previous research indicates that earlier and longer exposures to screen time are associated with increased risk of psychiatric conditions (i.e., attentional problems and hyperactivity, anxiety, and depression) $[19,39]$, further suggesting that Generation $\mathrm{Z}$ children would be more likely to exhibit learning and memory impairments when compared to Generation X children.

Excessive screen time was found to be associated with problems in meeting developmental milestones for motor skills, spatio-temporal abilities, problem solving and language acquisition [7]. Content, pace, and degree of exposure were associated with dysfunction in attention, learning, and memory for both infants and children $[6,13]$. For example, observational experiments in humans and animals determined that excessive sensory stimulation and exposure to fast-paced television during infancy and childhood resulted in cognitive and behavioural deficits [13]. Similarly, television programs which encourage active participation facilitated greater vocabulary acquisition when compared to passive programming [6]. A decline in performance on cognitive tasks was also observed in children who had over two hours of screen time per day and children exposed to seven 
or more hours of screen time per day revealed significantly reduced orbitofrontal thickness [32], a fundamental structure in the reward circuit pathway responsible for decisionmaking and addiction disorders in adolescence and adulthood $[25,31,96]$.

The consequences of digital technology are associated with prolonged effects in adulthood. For example, postsecondary students scored significantly higher on midterms and exams in classrooms that prohibited the use of electronic devices in comparison to classrooms that did not prohibit the use of electronic devices [9]. In the general population, long-term effects of excessive screen time were associated with reductions in long-term memory and cognitive development [9]. In addition, many adults rely on technology and smartphones as opposed to cultivating self-imposed analytical thinking [76]. For instance, three studies examining smartphone use and cognition determined that individuals who thought more intuitively were more likely to rely on their smartphones for information when compared to individuals who thought more analytically [76]. Since search engines allow easy access of information, users are more prone to remember where to locate a fact instead of remembering the fact itself [10]. This can be seen when dealing with difficult questions: users tend to think more about searching for solutions online as they have a decreased recollection of the information and an enhanced recollection for where to access it [10].

Excessive screen time involving task switching between multiple screens also has harmful effects on memory, especially explicit memory which consists of episodic and semantic memory [97]. Episodic memory involves the recollection of autobiographical events from the past occurring in specific spatial and temporal contexts [98]. However, an overuse of digital recording of events (e.g., digital pictures, videos, etc.) may result in a loss of episodic memories as one begins to rely on external storage for memory rather than encoding it in the brain [97]. In a study evaluating the effects of using social media to record or share one's experiences, it was found that participants who did not use social media consistently remembered their experiences more accurately than those who used social media [11]. These results suggest that using digital media may prevent people from remembering the very events they are attempting to preserve [11]. Semantic memory is described as the general knowledge and recall of factual information [98]. Information on the internet is often presented in hypertexts permitting users to scan information superficially which can lead to poor memory recollection [37]. Digital media adapts at a rapid pace, producing fundamental changes in written word or text (e.g., internet shorthand such as acronyms, keyboard symbols and abbreviations) [99]. Constant literary changes impede one's ability to internalize the categorical rules of language like verb conjugation, spelling, and punctuation. Today, penmanship is dramatically diminished, which may drastically impact future generations more significantly because it serves as a tool for learning and consolidating memories [99].

In a study that evaluated the concept of digital dementia, the starting age and amount of mobile device use were potential factors leading to cognitive decline in males and females either under 20 years old or over 21 [75]. Excessive screen time is also associated with decreased attention and memory ability, alongside a greater amount of stress endured [75]. Generation $\mathrm{Z}$ may be a high-risk population for ADRD due to their heavy reliance on technology during critical periods of brain development and maturation. Many symptoms of excessive screen time parallel early cognitive decline as language and memory are severely impaired.

\subsection{Effects of excessive screen time on attentional disorders}

The rise of infant television viewing started in the late 1990s and has only become progressively more common [100]. The United States has seen a 10-fold rise in the incidence of attention-deficit disorder diagnoses in the past 20 years [100]. Television exposure early on is associated with attentional problems; in one study, 10\% of children aged 1 to 3 developed attentional problems as early as the age of 7 [12]. Children viewed an average of 2.2 hours of television per day at age 1 and 3.6 hours per day at age 3 . A one point standard deviation increase in the number of hours of television watched at age 1 is linked with a $28 \%$ rise in the likelihood of having attentional problems at age 7 [12].

Furthermore, increased multitasking on digital devices, particularly during study time, increases the risk of poor academic progress for adolescents and young adults [9, 14]. Interestingly, another study proposing a brain drain hypothesis for the effects of digital technology on cognition demonstrated that even when people are successful at maintaining sustained attention and avoid checking their phones, the mere presence of these devices still reduces available cognitive capacity [16]. The presence of a smartphone can adversely affect two measures of cognitive capacity, working memory capacity and functional fluid intelligence; both were negatively affected in those who had the highest rates of smartphone dependence [16].

Lastly, "digital natives", such as Generation Z, are generations that grow up with internet technologies and drift towards a shallow information processing behavior distinguished by rapid attention shifting [37]. They also display higher incidence of internet-related addictive behaviors that reflect changed reward-processing and self-control mechanisms. Neuroimaging investigations have indicated associations between these internet-related cognitive effects and fundamental alterations in the brain [37]. A study that analyzed multitasking between different generations found that Millennials and Generation $\mathrm{Z}$ reported more multitasking than Generation X, who reported more multitasking than the Baby Boomers [14]. Generation Z differs significantly than Baby Boomers as they engage in heightened multitasking activities which are related to increased distractibility and poor executive control abilities [14]. Attention is necessary for all cognitive activities, and impairments in attention in adoles- 
cence and early adulthood that persist into middle and late adulthood may greatly reduce overall brain reserve leading to greater risk and early signs of MCI and ADRD [101, 102].

4.8 Effects of excessive screen time on social emotional functioning and self-care

Generation $\mathrm{Z}$ individuals see their friends in person an hour less a day than Millennials did at similar ages [41]. On average, Generation $\mathrm{Z}$ adolescents in Grade 12 spend approximately 2 hours a day texting, 2 hours a day on the internet (including gaming) and 2 hours a day on social media for a total of 6 hours a day of screen time [44]. When the smartphones became more available around 2010, fewer adolescents reported that they were happy [20]. Adolescents who scored high on-screen use were significantly more likely to display poor emotion regulation, an inability to stay calm, irritability, uncooperative attitudes, lower productivity and curiosity, and damaged sociability [20]. Poor mental health is correlated with excessive screen time (any more than 2 hours per day), less sleep, or decreased in-person interactions [44]. Excessive screen use contributes to a sedentary lifestyle which is associated with reduced physical activity, lower vascular fitness, and an increased incidence of being overweight or obese [40]. In a 2007 survey it was found that $20.8 \%$ of 6 to 11 -year olds and $26.1 \%$ of 12 to 17 -year olds engaged in excessive screen time. Having a TV in their bedroom, for both groups, was strongly associated with excessive screen time. For the older age group, it was also strongly associated with obesity [42]. Excessive screen time on social media has negative impacts on sleep duration and/or sleep quality [41]. Adolescents in 2015 were 16-17\% more likely to report sleeping less than 7 hours a night compared to those in 2009 [41]. Screen time, such as electronic device use, social media, and reading news online, rose during this time period [41]. This was linked with heightened odds of short sleep duration, with a clear exposure-response relationship for electronic devices after 2 or more hours of use per day [41]. Experimental studies show that limiting social media use to less than 30 minutes per day may lead to significant improvements in well-being (e.g., significant reduction in loneliness and depression) [38]. Symptoms of impaired self-care, emotional regulation, and social functioning are characteristic of MCI and ADRD [48]. Impairments in instrumental activities of daily living in adolescence and early adulthood that persist or worsen in middle adulthood could lead to diminished brain and cognitivebehavioural reserves increasing the risk of early onset dementia.

4.9 Effects of excessive screen time on the development of psychiatric disorders

Studies show that early sociobiological embedding of stressors tends to have enduring lifetime effects rather than transient ones and increases the risk of lifetime physical and mental illness and social problems [103]. Brain structure and function, which is the most malleable during development, is continually remodeled by environmental factors and is particularly sensitive to acute and chronic stressors which exert their effects through the neuro-immuno-endocrine system [103]. Although neuroplastic changes in response to stressors may confer adaptive advantages in the short-term (allostasis), the persistence of changes that are abnormal and irreversible can be maladaptive later in life under accumulating pressures of the biopsychosocial environment (allostatic load and overload) [103]. Excessive screen time can act as both a non-normative stimulus, exerting its effects at a sub-stress threshold level, and as an acute and chronic stressor, increasing the risk of psychiatric problems during development and later in life [4]. Evidence is emerging that excessive social media use is a significant psychosocial stressor contributing to mental health problems in adolescents and young adults, for example, by lowering global self-esteem and heightening the risk of internet addiction, and increasing the prevalence of psychopathology including attentional, hyperactivity, anxiety, and depressive disorders [4]. Unlike offline face-to-face social connection, online virtual social interaction is associated with greater incidence of internalizing problems such as social anxiety and depression [4, 20]. Specifically, the association between screen time and depression was proposed and tested with three explanatory hypotheses: displacement, upward social comparison, and reinforcing spirals [17]. Upward social comparison suggests that the impact of screen time on mental health depends on the kind of content being viewed; for example, when individuals compare themselves with others who they consider are in more favorable positions (e.g., those with perfect bodies and lives) [17]. Reinforcing spirals also posits that screen time effects are mediated through content; however, reinforcing spirals adds that individuals try to obtain and choose information consistent with their thoughts which may then become increasingly negative in nature [17]. The displacement hypothesis suggests that all screen time adversely affects mental health because it displaces time one could be engaging in physical exercise and other healthy behaviours [17]. It was found that the upward social comparison and reinforcing spirals hypotheses explained associations between social media, television, and depression more than the displacement hypothesis [17]. Since those on social media platforms share the most idealized versions of themselves, it becomes commonplace for individuals to compare their real-life experiences with those of others who highlight positive moments, causing deleterious effects on self-esteem and life satisfaction [17]. Of the four types of screen use assessed (i.e., social media, TV viewing, video gaming, and computer use), only greater levels of social media and computer use were associated with depression, but not TV viewing or video gaming [17]. However, in a study on the relationship between screen time and psychological distress, internet addiction was associated with greater online video gaming and sexual activity, higher anxiety levels, and low email use [23]. Individuals with internet addiction also had increased avoidance coping mechanisms and higher rumination whilst having worsened self-care behaviors, such as seeking social support and getting adequate sleep [23]. Screen 
time potentially worsens- or in some cases creates- mental health problems which some speculate may lead to premature neurodegeneration and increased risk of premature cognitive decline in later adulthood [4].

\subsection{Limitations of the hypothesis and counterarguments on the evidence}

Given the significant increases in average daily use of digital technology, we are concerned about the possibility of a rising trend in ADRD attributed to excessive screen time. To date, few studies have assessed the relationship between excessive screen time during development and ADRD, which is concerning because the ADRD burden is expected to at least double by 2060 [1]. The estimates provided in Matthews and colleagues (2019) [1] were from a nationally representative sample, ADRD was physician-diagnosed in a clinical setting, and sociodemographic characteristics (i.e., age, race, sex, and ethnicity) were included. We considered the same estimates as outlined in Matthews and colleagues [1] and also considered the effects of screen time and type, by age, and developmental stage. Statistical analyses by sex, race, or ethnicity were omitted because the data is not available or is not significant for these factors. Matthews and colleagues [1] also note that estimates did not include socioeconomic factors, such as education, which is a known risk factor in ADRD research [104]. To address this, our proposed hypothesis considered education attainment and IQ. Our report is the first to propose and explore the relationship between ADRD and screen time during development. Future research should consider additional factors, for example the serious short- and longterm implications of excessive screen time for the development of youth and young adult CBBR. These should include the short term implications of overall increased screen time from greater digital technology use both at home and at school (e.g., increased anxiety, depression, substance abuse, suicidal ideation and attempts etc.) and their long term consequences (e.g., permanent effects on brain development that will affect learning, memory and intelligence level, poorer school performance, greater risk of internalizing and externalizing psychological disorders, poorer overall health and economic outcomes, etc.). Future research should examine the lifelong implications of excessive screen time because it represents a significant proportion of daily activity during development, equal or greater than time spend on traditional educational activities (i.e., face-to-face instructional learning, reading from print, learning to hand write words and numbers). Additionally, physical activity should be included as a covariate because research shows that a reduction in physical activity is associated with increased screen time [105] and risk of developing ADRD [106-108].

While research on ADRD and screen time during development is limited, the current literature indicates that cognitive activity and physical activity are the two major modifiable risk factors for ADRD [106] and both cognitive and physical activity have also shown associations with screen time [109]. However, counterarguments on the evidence are mixed and inconclusive as to whether brain structure and physiology are associated with cognitive activity [106]. For example, cognitive activities may cross domains (i.e., completing a puzzle with a friend is both a cognitive activity and a social activity) and no such methods exist to classify the rate of cognitive activities (i.e., high cognitive activity versus a low cognitive activity), introducing noise into the variable [106]. Prospective research should continue to monitor the longterm effects of excessive screen time, including the ABCD study [33] that records the amount of screen use, amongst other factors, and the incidence of dementia in a large population in 2060. Preliminary results, for example in 2020 and 2040, could help inform future health policy decisions and potential preventative actions. Ideally research should focus on a number of factors including the influence of duration and type of media in order to inform appropriate guidelines and recommendations. A predicted effective approach would be to focus on informing the public on the dangers of excessive screen time use.

\section{Consequences of the hypothesis and discussion}

The projected estimates of those with ADRD, as reported in Matthews and colleagues (2019) [1], will double from 1.6\% to $3.3 \%$, meaning 13.9 million Americans are predicted to have ADRD in 2060. We, however, predict that from in 2060 to 2100 , the rates of ADRD will rise far above the CDC's projected estimates of a 2-fold increase to up to a four-to six-fold increase. With our prediction this would mean that up to 26.7 to 48.0 million Americans aged $\geq 65$ years could potentially show signs of ADRD between 2060 and 2100 as Millennials and Generation $\mathrm{Z}$ become the majority of the elderly population. In minority populations, the disproportionate prevalence of ADRD could amplify current socio-economic disparities leading to worse health outcomes for those with ADRD and their subsequent caregivers [1]. If our proposed hypothesis is correct, then there are substantial consequences to excessive screen time for Millenials and Generation Z, and future generations to follow, which include their children and grandchildren. There are three main courses of action available to address the implications of our hypothesis: treatment, mitigation, and prevention. Perhaps the easiest, but most costly, will be treatment, whereas the least costly but most difficult will be prevention. As such, mitigation may be the most pragmatic and effective overall.

First and foremost, for the majority of Millenials and Generation $\mathrm{Z}$, the negative effects of excessive screen time on development and cognitive-behavioural-brain reserve have already occurred. Thus, any negative effects can only be treated in late adulthood or potentially mitigated before then. It is possible for the youngest Generation $\mathrm{Z}$ individuals that these effects could potentially be reversed with targeted intervention. If nothing is done to address the concerns raised here over the next 40 years, then treatment of MCI and ADRD will be the main course of action. The health care system is 
not prepared to handle the future social and economic burden of an ADRD patient population estimated to more than double or triple in size by 2060 . For example, the ratio of potential caregivers to patients is currently 7 -to- 1 for adults in the high-risk age group, but this support is anticipated to decrease to 4-to-1 by 2030 [110]. If our projected estimates are correct it will be far lower by 2060. Furthermore, caregivers often report experiencing work disruption, decreased income and personal savings, poorer physical health increased rates of psychological distress and depression, and have increased mortality risks [111-113]. The full and future socioeconomic costs to society of ADRD are challenging to gauge, specifically in light of new and converging data suggesting that factors unique to youth and young adults today, such as declining global IQ and rapidly increasing rates of screen time, may increase their risk of accelerated neurodegeneration in early and middle adulthood and rates of ADRD in late adulthood. Recent estimates of the worldwide costs of ADRD in 2015 were equivalent to $1.1 \%$ of the global gross domestic product (GDP) and more than $85 \%$ of these costs occur in highincome countries such as the US and Canada [114]. Global economic costs of ADRD are projected to double from $\$ 1$ trillion to $\$ 2$ trillion (USD) in the next decade alone (e.g., from 2018 to 2030) [114, 115]. If our predictions are correct, and the prevalence of ADRD will increase by more than double the current projected rates estimated by Matthews and colleagues [1], this could cripple or even collapse the healthcare systems in North America.

Second, significant efforts could be made to mitigate the negative effects of screen time for Millenial and Generation $\mathrm{Z}$ adults with interventions targeted at reducing screen time, treating physical and mental health problems associated with screen time during early adulthood, and promoting factors known to increase cognitive-behavioural-brain reserve. Proper management of health, lifestyle, and wellness choices can reduce the risk of ADRD. A significant reduction in screen time will help mitigate the incidence of ADRD through preventing cognitive impairment. For example, correlational and experimental studies show that reducing screen time can improve concentration, learning, and memory $[9,12,116]$, psychological well-being [117-119], reduce experiences of anxiousness and depressed moods [120], improve sleep [121] and overall mental health [63]. Studies in humans and animals models show that environmental enrichment can reduce or reverse some of the effects of deprivation, non-normative stimulation and even acute or chronic stress [122,123] at the behavioural and neural levels [109, 124-126]. Evidence suggests that brain structure volume is influenced by environmental enrichment [106]. For instance, the London taxi driver study found that taxi drivers who drove passively (i.e., following fixed routes) had more prominent signs of hippocampal atrophy when compared to taxi drivers who drove actively (i.e., driving routes exclusively from memory) [127]. The latter raises the question as to whether students who passively take notes (i.e., on a lap- top or tablet) may be more at risk for ADRD than students who take notes actively (i.e., longhand) [109], given that active participation increases cognitive-behavioural-brain reserve [106]. Additionally, increasing physical activity can prevent ADRD by improving cognitive reserve [106]. Physical activity, defined as 20 minutes of high-intensity training for 3 or more days per week or moderate-intensity training for 5 or more days per week, has been shown to decrease hippocampal, prefrontal and cingulate cortex atrophy $[128,129]$, all hallmarks of ADRD. By minimizing screen time, physical and mental health and environmental enrichment will improve, reducing the risk of ADRD. Correspondingly, improving physical and mental health and encouraging environmental enrichment can help to minimize screen time, and in turn lower the risk of ADRD. Thus, highlighting that the relationship between ADRD and screen time, physical and mental health, and cognitive reserve is bidirectional (i.e., improving one variable, directly affects another variable, and vice versa).

Last but not least, for future generations, it is still possible to completely avoid the detrimental effects of screen time and thus preventive measures should be the predominant focus. Excessive screen time is associated with negative physical, mental and social health effects as well as learning and behavioral disadvantages; as such potentially drastic measures should be implemented to decrease both the duration and consistency of screen use. For example, public and private investments (i.e., scholarships, awards, bursaries, and grants) could be used to encourage students from elementary through post-secondary school to replace digital learning with traditional learning. Students who can demonstrate more time engaging in non-digital enrichment activities when compared to digital enrichment activities can be eligible to apply for or receive monetary compensation. Data shows that institutional aid motivates students and encourages persistence towards program completion and graduation [130]. Additionally, education should be created to focus more on enriched environments promoting spatio-temporal learning and higher order analytic abilities, which both contribute to minimizing the risk of developing ADRD $[7,106]$. Legislature can be implemented such that there are legal and economic costs (i.e., fines and incarceration) for individuals/companies who exploit the addictive nature of technology. Penalties should be of similar nature to how the US government prosecuted and convicted Big Tobacco companies for deceiving the public and racketeering [131]. Also, digital-free zones and mass public education campaigns can be implemented in public places such as schools, workplaces, places of worship, shopping malls, and restaurants with signs that promote physical activity and environmental enrichment, both proven to reduce the risk of ADRD [106]. In the short-term, the implementation of these measures will assist with reducing screen time, improve sleep and overall mental health, and have long-term implications for preventing ADRD. 


\section{Summary and conclusions}

Estimates of the projected 2-to-4-fold increase in ADRDs for 2060 are based on age, sex, race, and ethnicity, and do not take into account significant and relevant differences in the populations used to make these predictions (i.e., Silent Generation and Baby Boomers) and the populations who will be represented in this growing burden of disease in 2060 (i.e., Millenials and Generation Z). We have identified two known critical factors that differ significantly between individuals born before 1965 and those born after 1980 which are related to the risk of MCI and ADRD: excessive screen time and intelligence. Excessive screen time has been empirically demonstrated to affect brain development and increase the risk of cognitive, emotional, and behavioural disorders in adolescents and young adults by negatively impacting attention and concentration, learning and memory, emotional regulation and social functioning, physical health, and development of mental disorders and substance use. These effects are similar to the symptoms of MCI seen in older adults that increase the risk of ADRDs. The current decline in global intelligence, estimated to have started post-1975 and projected to continue into 2050, parallels significant population level increases in screen time and could also increase the risk of MCI and ADRD. We have provided a detailed model of the effects of excessive screen time on CBBR expected to contribute to the increased risk of MCI and ADRD in Millenials and Generation $\mathrm{Z}$ in 2060 and beyond. The CBBR hypothesis of dementia postulates that more complex patterns of neural and mental activity in early, middle, and later life stages are associated with decreased risk of dementia as opposed to less complex patterns which are associated with increased risks. Studies show that these cortical networks and associated cognitive-behavioural abilities can be influenced by early environmental experiences including excessive screen time. If the neural circuits underlying these cognitive-behavioural abilities essential for general intelligence and lifetime adaptability are under- or abnormally-developed before adulthood, then it is likely that these changes will persist into early and middle adulthood and be more vulnerable to accelerated neurodegeneration in late adulthood therefore increasing the risk of early onset MCI and ADRDs. We present three main courses of action focused on treatment, mitigation, and prevention as measures to counteract the potential 4-to-6-fold increase in rates of ADRD post-2060 which could result in widespread societal and economic distress in an already overburdened healthcare system in North America.

\section{Abbreviations}

$\mathrm{ABCD}$, Adolescent Brain and Cognitive Development; ADRD, Alzheimer's disease and related dementias; CBBR, cognitive-behavioural brain reserve; CDC, Centres for Disease Control; GDP, gross domestic product; IQ, intelligence quotient; MCI, mild cognitive impairment; MDD, mobile digital devices.

\section{Author contributions}

LAM conceived and designed the study; LAM, MT, TMC, RE contributed to analyzing the data and writing the manuscript; LAM added graphics and edited the manuscript.

\section{Ethics approval and consent to participate}

Not applicable.

\section{Acknowledgment}

We wish to thank the two anonymous reviewers for their excellent criticism of the article and suggestions to improve its quality.

\section{Funding}

This research received no external funding.

\section{Conflict of interest}

The authors declare no conflict of interest.

\section{References}

[1] Matthews KA, Xu W, Gaglioti AH, Holt JB, Croft JB, Mack D, et al. Racial and ethnic estimates of Alzheimer's disease and related dementias in the United States (2015-2060) in adults aged $\geq 65$ years. Alzheimer's \& Dementia. 2019; 15: 17-24.

[2] Lynn R, Harvey J. The decline of the world's IQ. Intelligence. 2008; 36: $112-120$.

[3] Bratsberg B, Rogeberg O. Flynn effect and its reversal are both environmentally caused. Proceedings of the National Academy of Sciences. 2018; 115: 6674-6678.

[4] Neophytou E, Manwell LA, Eikelboom R. Effects of Excessive Screen Time on Neurodevelopment, Learning, Memory, Mental Health, and Neurodegeneration: a Scoping Review. International Journal of Mental Health and Addiction. 2019; 19: 724-744.

[5] Valenzuela MJ, Sachdev P. Brain reserve and dementia: a systematic review. Psychological Medicine. 2005; 36: 441-454.

[6] Linebarger DL, Walker D. Infants' and Toddlers' Television Viewing and Language Outcomes. American Behavioral Scientist. 2005; 48: 624-645.

[7] Madigan S, Browne D, Racine N, Mori C, Tough S. Association between Screen Time and Children's Performance on a Developmental Screening Test. American Medical Association. 2019; 173: 244-250.

[8] Mangen A, Walgermo BR, Brønnick K. Reading linear texts on paper versus computer screen: Effects on reading comprehension. International Journal of Educational Research. 2013; 58: 61-68.

[9] Glass AL, Kang M. Dividing attention in the classroom reduces exam performance. Educational Psychology. 2019; 39: 395-408.

[10] Sparrow B, Liu J, Wegner DM. Google effects on memory: cognitive consequences of having information at our fingertips. Science. 2011; 333: 776-778.

[11] Tamir DI, Templeton EM, Ward AF, Zaki J. Media usage diminishes memory for experiences. Journal of Experimental Social Psychology. 2018; 76: 161-168.

[12] Christakis DA, Zimmerman FJ, DiGiuseppe DL, McCarty CA. Early television exposure and subsequent attentional problems in children. Pediatrics. 2004; 113: 708-713.

[13] Christakis DA, Ramirez JSB, Ferguson SM, Ravinder S, Ramirez J. How early media exposure may affect cognitive function: a review of results from observations in humans and experiments in mice. Proceedings of the National Academy of Sciences of the United States of America. 2018; 115: 9851-9858.

[14] Carrier LM, Cheever NA, Rosen LD, Benitez S, Chang J. Multitasking across generations: Multitasking choices and difficulty 
ratings in three generations of Americans. Computers in Human Behavior. 2009; 25: 483-489.

[15] Hartanto A, Yang H. Is the smartphone a smart choice? The effect of smartphone separation on executive functions. Computers in Human Behavior. 2016; 64: 329-336.

[16] Ward AF, Duke K, Gneezy A, Bos MW. Brain Drain: the Mere Presence of one's Own Smartphone Reduces Available Cognitive Capacity. Journal of the Association for Consumer Research. 2017; 2: 140-154.

[17] Boers E, Afzali MH, Newton N, Conrod P. Association of Screen Time and Depression in Adolescence. Journal of Mental Health and Addiction. 2019; 173: 853-859.

[18] Lepp A, Barkley JE, Karpinski AC. The relationship between cell phone use, academic performance, anxiety, and Satisfaction with Life in college students. Computers in Human Behavior. 2014; 31 : 343-350.

[19] Maras D, Flament MF, Murray M, Buchholz A, Henderson KA, Obeid N, et al. Screen time is associated with depression and anxiety in Canadian youth. Preventive Medicine. 2015; 73: 133-138.

[20] Twenge JM, Campbell WK. Associations between screen time and lower psychological well-being among children and adolescents: Evidence from a population-based study. Preventive Medicine Reports. 2018; 12: 271-283.

[21] Twenge JM, Cooper AB, Joiner TE, Duffy ME, Binau SG. Age, period, and cohort trends in mood disorder indicators and suiciderelated outcomes in a nationally representative dataset, 20052017. Journal of Abnormal Psychology. 2019; 128: 185-199.

[22] Kolb B, Wishaw IQ. Fundamentals of Neuropsychology. 7th edn. Worth Publishers: New York. 2015

[23] McNicol ML, Thorsteinsson EB. Internet Addiction, Psychological Distress, and Coping Responses among Adolescents and Adults. Cyberpsychology, Behavior and Social Networking. 2017; 20: 296-303.

[24] Selfhout MHW, Branje SJT, Delsing M, ter Bogt TFM, Meeus WHJ. Different types of Internet use, depression, and social anxiety: the role of perceived friendship quality. Journal of Adolescence. 2009; 32: 819-833.

[25] Gommans R, Stevens GWJM, Finne E, Cillessen AHN, BonielNissim M, Ter-Bogt TFM. Frequent electronic media communication with friends is associated with higher adolescent substance use. International Journal of Public Health. 2015; 60: 167-177.

[26] Hall W, Degenhardt L, Teesson M. Understanding comorbidity between substance use, anxiety and affective disorders: broadening the research base. Addictive Behaviors. 2009; 34: 526-530.

[27] Hulse GK, Lautenschalger NT, Tait RJ, Almeida O. Dementia associated with alcohol and other drug use. International Psychogeriatrics. 2005; 17: S109-S127.

[28] Zilkens R, Bruce D, Duke J, Spilsbury K, Semmens J. Severe Psychiatric Disorders in Mid-Life and Risk of Dementia in Late-Life (Age 65-84 Years): a Population Based Case-Control Study. Current Alzheimer Research. 2014; 11: 681-693.

[29] He Q, Turel O, Brevers D, Bechara A. Excess social media use in normal populations is associated with amygdala-striatal but not with prefrontal morphology. Psychiatry Research. Neuroimaging. 2017; 269: 31-35.

[30] Hong S, Zalesky A, Cocchi L, Fornito A, Choi E, Kim H, et al. Decreased functional brain connectivity in adolescents with internet addiction. PLoS ONE. 2013; 8: e57831.

[31] Hong SB, Kim JW, Choi EJ, Kim HH, Suh JE, Kim CD, et al. Reduced orbitofrontal cortical thickness in male adolescents with internet addiction. Behavioral and Brain Functions. 2013; 9: 1-5.

[32] Lee L. Screen time changes structure of kids' brains. "60 minutes" says. 2018. Available at: https://www.cbsnews.com/news/gro undbreaking-study-examines-effects-of-screen-time-on-kid s-60-minutes/ (Accessed: 19 September 2021).

[33] Paulus MP, Squeglia LM, Bagot K, Jacobus J, Kuplicki R, Breslin FJ, et al. Screen media activity and brain structure in youth: Evidence for diverse structural correlation networks from the ABCD study. NeuroImage. 2019; 185: 140-153.
[34] Weng C, Qian R, Fu X, Lin B, Han X, Niu C, et al. Gray matter and white matter abnormalities in online game addiction. European Journal of Radiology. 2013; 82: 1308-1312.

[35] Yuan K, Qin W, Wang G, Zeng F, Zhao L, Yang X, et al. Microstructure abnormalities in adolescents with internet addiction disorder. PLoS ONE. 2011; 6: e20708.

[36] Yuan K, Cheng P, Dong T, Bi Y, Xing L, Yu D, et al. Cortical thickness abnormalities in late adolescence with online gaming addiction. PLoS ONE. 2013; 8: e53055.

[37] Loh KK, Kanai R. How has the Internet Reshaped Human Cognition? The Neuroscientist: a Review Journal Bringing Neurobiology, Neurology and Psychiatry. 2016; 22: 506-520.

[38] Hunt MG, Marx R, Lipson C, Young J. No more FOMO: Limiting social media decreases loneliness and depression. Journal of Social and Clinical Psychology. 2018; 37: 751-768.

[39] Yen J, Yen C, Chen C, Tang T, Ko C. The Association between Adult ADHD Symptoms and Internet Addiction among College Students: the Gender Difference. CyberPsychology \& Behavior. 2009; 12: 187-191.

[40] Martin K. Electronic Overload: The Impact of Excessive Screen Use on Child and Adolescent Health and Wellbeing. 2011. Available at: https://www.natureplaywa.org.au/library/1/file/R esources/research/K\%20Martin\%202011\%20Electronic\%20Overl oad\%20DSR\%20(2).pdf (Accessed: 24 March 2011).

[41] Twenge JM, Krizan Z, Hisler G. Decreases in self-reported sleep duration among U.S. adolescents 2009-2015 and association with new media screen time. Sleep Medicine. 2017; 39: 47-53.

[42] Wethington H, Pan L, Sherry B. The association of screen time, television in the bedroom, and obesity among school-aged youth: 2007 national survey of children's health. Journal of School Health. 2013; 83: 573-581.

[43] Twenge JM, Campbell WK, Sherman RA. Declines in vocabulary among American adults within levels of educational attainment (1974-2016). Intelligence. 2019; 76: 101377.

[44] Twenge JM, Martin GN, Spitzberg BH. Trends in US Adolescents' media use: The rise of digital media, the decline of TV, and the (near) demise of print. Psychology of Popular Media Culture. 2019; 8: 329-345.

[45] Thambisetty M. Mild cognitive impairment: An update for the practicing clinician. BMH Medical Journal. 2020; 7.

[46] Sanders S, Morano C. Alzheimer's disease and related dementias. Journal of Gerontological Social Work. 2008; 50: 191-214.

[47] Jeste DV, Finkel SI. Psychosis of Alzheimer's disease and related dementias. Diagnostic criteria for a distinct syndrome. American Journal of Geriatric Psychiatry. 2000; 8: 29-34.

[48] Porter VR, Buxton WG, Fairbanks LA, Strickland T, O'Connor SM, Rosenberg-Thompson S, et al. Frequency and characteristics of anxiety among patients with Alzheimer's disease and related dementias. Journal of Neuropsychiatry and Clinical Neurosciences. 2003; 15: 180-186.

[49] Shi L, Chen S, Ma M, Bao Y, Han Y, Wang Y, et al. Sleep disturbances increase the risk of dementia: a systematic review and meta-analysis. Sleep Medicine Reviews. 2018; 40: 4-16.

[50] Anderson VM, Schott JM, Bartlett JW, Leung KK, Miller DH, Fox NC. Gray matter atrophy rate as a marker of disease progression in AD. Neurobiology of Aging. 2012; 33: 1194-1202.

[51] Avants BB, Cook PA, Ungar L, Gee JC, Grossman M. Dementia induces correlated reductions in white matter integrity and cortical thickness: a multivariate neuroimaging study with sparse canonical correlation analysis. NeuroImage. 2010; 50: 1004-1016.

[52] Fox NC, Crum WR, Scahill RI, Stevens JM, Janssen JC, Rossor MN. Imaging of onset and progression of Alzheimer's disease with voxel-compression mapping of serial magnetic resonance images. Lancet. 2001; 358: 201-205.

[53] Jack CR, Shiung MM, Gunter JL, O’Brien PC, Weigand SD, Knopman DS, et al. Comparison of different MRI brain atrophy rate measures with clinical disease progression in AD. Neurology. 2004; 62: 591-600.

[54] Scahill RI, Schott JM, Stevens JM, Rossor MN, Fox NC. Mapping 
the evolution of regional atrophy in Alzheimer's disease: unbiased analysis of fluid-registered serial MRI. Proceedings of the National Academy of Sciences of the United States of America. 2002; 99: 4703-4707.

[55] Stout JC, Jernigan TL, Archibald SL, Salmon DP. Association of dementia severity with cortical gray matter and abnormal white matter volumes in dementia of the Alzheimer type. Archives of Neurology. 1996; 53: 742-749.

[56] Lin F, Zhou Y, Du Y, Qin L, Zhao Z, Xu J, et al. Abnormal white matter integrity in adolescents with internet addiction disorder: a tract-based spatial statistics study. PLoS ONE. 2012; 7: e30253.

[57] Lehmann M, Rohrer JD, Clarkson MJ, Ridgway GR, Scahill RI, Modat M, et al. Reduced Cortical Thickness in the Posterior Cingulate Gyrus is Characteristic of both Typical and Atypical Alzheimer's Disease. Journal of Alzheimer's Diseases. 2010; 20: 587-598.

[58] Dutton E, Van Der Linden D, Lynn R. The negative Flynn effect: A systematic review. Intelligence. 2016; 59: 163-169.

[59] Flynn JR. The mean IQ of Americans: Massive gains 1932 to 1978. Psychological Bulletin. 1984; 95: 29-51.

[60] Flynn JR. Massive IQ gains in 14 nations: What IQ tests really measure. Psychological bulletin, 1987; 101: 171-191.

[61] Flynn JR, Rossi-Casé L. IQ gains in Argentina between 1964 and 1998. Intelligence. 2012; 40: 145-150.

[62] Pietschnig J, Voracek M. One Century of Global IQ Gains: a Formal Meta-Analysis of the Flynn Effect (1909-2013) Perspectives on Psychological Science. 2015; 10: 282-306.

[63] Twenge JM. iGen: Why Today's Super-Connected Kids are Growing Up Less Rebellious, More Tolerant, Less Happy and Completely Unprepared for Adulthood (and What This Means for the Rest of Us). Atria Books: New York. 2017.

[64] Viens A. Visualizing Social Media Use by Generation. 2019. Available at: https://www.visualcapitalist.com/visualizing-social-med ia-use-by-generation/ (Accessed: 21 September 2019).

[65] Andò R, Pizza S, Corsini F. Watching television today. A comparative survey of Italian and American students' habits in front of television. Journal of Italian Cinema \& Media Studies. 2016; 4: 283-306.

[66] Dias P. Motivations for multi-screening: an exploratory study on motivations and gratifications. European Journal of Communication. 2016; 31: 678-693.

[67] Marinelli A, Andò R. Multiscreening and social TV. Journal of European Television History and Culture. 2014; 3: 24-36.

[68] McGill M, Williamson JH, Brewster SA. A review of collocated multi-user TV. Personal and Ubiquitous Computing. 2015; 19: 743-759.

[69] Angell R, Gorton M, Sauer J, Bottomley P, White J. Don't Distract me when I'm Media Multitasking: toward a Theory for Raising Advertising Recall and Recognition. Journal of Advertising. 2016; 45: 198-210.

[70] Jeong S, Hwang Y. Media Multitasking Effects on Cognitive vs. Attitudinal Outcomes: a Meta-Analysis. Human Communication Research. 2016; 42: 599-618.

[71] Kazakova S, Cauberghe V, Pandelaere M, De Pelsmacker P. Can't See the Forest for the Trees? The Effect of Media Multitasking on Cognitive Processing Style. Media Psychology. 2015; 18: 425-450.

[72] Segijn CM, Voorveld HAM, Smit EG. The underlying mechanisms of multiscreening. Journal of Advertising. 2016; 45: 391402 .

[73] Segijn CM, Voorveld HM, Vandeberg L, Smit EG. The battle of screens: Unraveling attention allocation and memory effects when multiscreening. Human Communication Research. 2017; 43: 295314.

[74] Collins A, Koechlin E. Reasoning, learning, and creativity: frontal lobe function and human decision-making. PLoS Biology. 2012; 10: e1001293.

[75] Yamamoto H, Ito K, Honda C, Aramaki E. Does digital dementia exist? California: Spring Symposium. 2018.

[76] Barr N, Pennycook G, Stolz JA, Fugelsang JA. The brain in your pocket: Evidence that Smartphones are used to supplant thinking. Computers in Human Behavior. 2015; 48: 473-480.

[77] Andersen SL. Trajectories of brain development: point of vulnerability or window of opportunity? Neuroscience and Biobehavioral Reviews. 2003; 27: 3-18.

[78] Giedd JN, Raznahan A, Alexander-Bloch A, Schmitt E, Gogtay N, Rapoport JL. Child psychiatry branch of the National Institute of Mental Health longitudinal structural magnetic resonance imaging study of human brain development. Neuropsychopharmacology. 2015; 40: 43-49.

[79] Kolb B, Whishaw IQ. Brain plasticity and behavior. Annual Review of Psychology. 1998; 49: 43-64.

[80] Geng X, Li G, Lu Z, Gao W, Wang L, Shen D, et al. Structural and Maturational Covariance in Early Childhood Brain Development. Cerebral Cortex. 2017; 27: 1795-1807.

[81] Saggar M, Hosseini SMH, Bruno JL, Quintin E, Raman MM, Kesler SR, et al. Estimating individual contribution from groupbased structural correlation networks. NeuroImage. 2015; 120 : 274-284.

[82] Van Den Heuvel MP, Sporns O. Network hubs in the human brain. Trends in Cognitive Sciences. 2013; 17: 683-696.

[83] Gold AL, Sheridan MA, Peverill M, Busso DS, Lambert HK, Alves $\mathrm{S}$, et al. Childhood abuse and reduced cortical thickness in brain regions involved in emotional processing. Journal of Child Psychology and Psychiatry. 2016; 57: 1154-1164.

[84] Besteher B, Gaser C, Spalthoff R, Nenadić I. Associations between urban upbringing and cortical thickness and gyrification. Journal of Psychiatric Research. 2017; 95: 114-120.

[85] Barch DM, Albaugh MD, Avenevoli S, Chang L, Clark DB, Glantz $\mathrm{MD}$, et al. Demographic, physical and mental health assessments in the adolescent brain and cognitive development study: Rationale and description. Developmental Cognitive Neuroscience. 2018; 32: 55-66.

[86] Bechara A. Decision making, impulse control and loss of willpower to resist drugs: a neurocognitive perspective. Nature Neuroscience. 2005; 8: 1458-1463.

[87] Zhou Y, Lin F, Du Y, Qin L, Zhao Z, Xu J, et al. Gray matter abnormalities in Internet addiction: a voxel-based morphometry study. European Journal of Radiology. 2011; 79: 92-95.

[88] Dong G, Devito EE, Du X, Cui Z. Impaired inhibitory control in 'internet addiction disorder': a functional magnetic resonance imaging study. Psychiatry Research. 2012; 203: 153-158.

[89] Dong G, Hu Y, Lin X. Reward/punishment sensitivities among internet addicts: Implications for their addictive behaviors. Progress in Neuro-Psychopharmacology \& Biological Psychiatry. 2013; 46: 139-145.

[90] Allegri RF, Taragano FE, Krupitzki H, Serrano CM, Dillon C, Sarasola D, et al. Role of cognitive reserve in progression from mild cognitive impairment to dementia. Dementia \& Neuropsychologia. 2010; 4: 28-34.

[91] Spitzer M. Digitale Demenz. Nervenheilkunde. 2012; 31: $493-$ 497.

[92] Gajewski RR. Pitfalls of E-education: From multimedia to digital dementia? Proceedings of the 2016 Federated Conference on Computer Science and Information Systems. 2016; 8: 913-920.

[93] Moledina S, Khoja A. Letter to the Editor: Digital Dementia-is Smart Technology Making us Dumb? Ochsner Journal. 2018; 18: 12.

[94] Zimmerman FJ, Christakis DA, Meltzoff AN. Television and DVD/video viewing in children younger than 2 years. Archives of Pediatrics \& Adolescent Medicine. 2007; 161: 473-479.

[95] Christakis DA, Zimmerman FJ. The Elephant in the Living Room: Make Television Work for Your Kids. Rodale Books: USA. 2006.

[96] Purcell K. 10 things to know about how teens use technology. 2013. Available at: https://www.pewinternet.org/2013/ 07/10/10-things-to-know-about-how-teens-usetechnology/ (Accessed: 10 July 2013).

[97] Van Dijck J. Mediated Memories in the Digital Age. Stanford University Press: USA. 2007. 
[98] Squire LR, Zola SM. Episodic memory, semantic memory, and amnesia. Hippocampus. 1998; 8: 205-211.

[99] O'Gorman M. Taking care of digital dementia. 2015. Available at: https://journals.uvic.ca/index.php/ctheory/article/view/ 15128 (Accessed: 18 February 2015).

[100] Christakis DA. The effects of infant media usage: what do we know and what should we learn? Acta Paediatrica. 2009; 98: 816.

[101] Balota DA, Faust M. Attention in dementia of the Alzheimer's type. Handbook of Neuropsychology. 2001; 6: 51-80.

[102] Xie H, Mayo N, Koski L. Predictors of Future Cognitive Decline in Persons with Mild Cognitive Impairment. Dementia and Geriatric Cognitive Disorders. 2011; 32: 308-317.

[103] McEwen BS. Brain on stress: how the social environment gets under the skin. Proceedings of the National Academy of Sciences. 2012; 109: 17180-17185.

[104] Sharp ES, Gatz M. The relationship between education and dementia an updated systematic review. Alzheimer Disease and Associated Disorders. 2011; 25: 289-304.

[105] Laurson KR, Eisenmann JC, Welk GJ, Wickel EE, Gentile DA, Walsh DA. Combined influence of physical activity and screen time recommendations on childhood overweight. Journal of Pediatrics. 2008; 153: 209-214.

[106] Cheng ST. Cognitive reserve and the prevention of dementia: the role of physical and cognitive activities. Current Psychiatry Reports. 2016; 18: 85.

[107] Kempermann G, Fabel K, Ehninger D, Babu H, Leal-Galicia $\mathrm{P}$, Garthe A, et al. Why and how physical activity promotes experience-induced brain plasticity. Frontiers in Neuroscience. 2010; 4: 189.

[108] Churchill JD, Galvez R, Colcombe S, Swain RA, Kramer AF, Greenough WT. Exercise, experience and the aging brain. Neurobiology of Aging. 2002; 23: 941-955.

[109] Mueller PA, Oppenheimer DM. The pen is mightier than the keyboard: Advantages of longhand over laptop note taking. Psychological Science. 2014; 25: 1159-1168.

[110] Redfoot D, Feinberg L, Houser A. The Aging of the Baby Boom and Growing Care Gap: A Look at Future Declines in the Availability of Family Caregivers. AARP Public Policy Institute: Washington, D.C. 2013.

[111] Schulz R, Beach SR. Caregiving as a Risk Factor for Mortality: the Caregiver Health Effects Study. Journal of Mental Health and Addiction. 1999; 282: 2215-2219.

[112] Sörensen S, Duberstein P, Gill D, Pinquart M. Dementia care: mental health effects, intervention strategies, and clinical implications. The Lancet Neurology. 2006; 5: 961-973.

[113] Vitaliano PP, Zhang J, Scanlan JM. Is caregiving hazardous to one's physical health? A meta-analysis. Psychological Bulletin. 2003: 129: 946

[114] Wimo A, Guerchet M, Ali GC, Wu YT, Prina AM, Winblad B, et al. The worldwide costs of dementia 2015 and comparisons with 2020. Alzheimer's and Dementia. 2017; 13: 1-7.

[115] El-Hayek YH, Wiley RE, Khoury CP, Daya RP, Ballard C, Evans AR, et al. Tip of the Iceberg: Assessing the Global Socioeconomic
Costs of Alzheimer's Disease and Related Dementias and Strategic Implications for Stakeholders. Journal of Alzheimer's Diseases. 2019; 70: 323-341.

[116] Christakis DA. The effects of infant media usage: what do we know and what should we learn. Acta Paediatrica. 2008; 98: 8-16.

[117] Shakya HB, Christakis NA. Association of Facebook use with compromised well-being: a longitudinal study. American Journal of Epidemiology. 2017; 18: 203-211.

[118] Tromholt M. The Facebook experiment: Quitting Facebook leads to higher levels of well-being. Cyberpsychology, Behavior and Social Networking. 2016; 19: 661-666.

[119] Kross E, Verduyn P, Demiralp E, Park J, Lee DS, Lin N, et al. Facebook use predicts declines in subjective well-being in young adults. PLoS ONE. 2013; 8: e69841.

[120] Twenge JM. The age of anxiety? Birth cohort changes in anxiety and neuroticism, 1952-1993. Journal of Personality and Social Psychology. 2000; 79: 1007-1021.

[121] Perrault AA, Bayer L, Peuvrier M, Afyouni A, Ghisletta P, Brockmann C, et al. Reducing the use of screen electronic devices in the evening is associated with improved sleep and daytime vigilance in adolescents. Sleep. 2019; 42: 125.

[122] Menezes J, Das Neves BHS, Gonçalves R, Benetti F, MelloCarpes PB. Maternal deprivation impairs memory and cognitive flexibility, effect that is avoided by environmental enrichment. Behavioural Brain Research. 2020; 381: 112468.

[123] Francis DD, Diorio J, Plotsky PM, Meaney MJ. Environmental enrichment reverses the effects of maternal separation on stress reactivity. Journal of Neuroscience. 2002; 22: 7840-7843.

[124] Yeykelis L, Cummings JJ, Reeves B. Multitasking on a single device: Arousal and the frequency, anticipation, and prediction of switching between media content on a computer. Journal of Communication. 2014; 64: 167-192.

[125] Christakis DA, Ramirez JSB, Ramirez JM. Overstimulation of newborn mice leads to behavioral differences and deficits in cognitive performance. Scientific Reports. 2012; 2: 1-6.

[126] Smoker TJ, Murphy CE, Rockwell AK. Comparing memory for handwriting versus typing. Proceedings of the Human Factors and Ergonomics Society. 2009; 53: 1744-1747.

[127] Maguire EA, Gadian DG, Johnsrude IS, Good CD, Ashburner J, Frackowiak RS, et al. Navigation-related structural change in the hippocampi of taxi drivers. Proceedings of the National Academy of Sciences. 2000; 97: 4398-4403.

[128] Ruscheweyh R, Willemer C, Krüger K, Duning T, Warnecke T, Sommer J, et al. Physical activity and memory functions: an interventional study. Neurobiology of Aging. 2011; 32: 1304-1319.

[129] Colcombe SJ, Erickson KI, Scalf PE, Kim JS, Prakash R, McAuley $\mathrm{E}$, et al. Aerobic exercise training increases brain volume in aging humans. Journals of Gerontology. 2006; 61: 1166-1170.

[130] Robb CA, Moody B, Abdel-Ghany M. College student persistence to degree: The burden of debt. Journal of College Student Retention: Research, Theory \& Practice. 2012; 13: 431-456.

[131] Botvin GJ. The Health Consequences of Smoking-50 Years of Progress: A Report of the Surgeon General. CDC Web Archive: USA. 2014. 